\title{
OPTIMIZING SOC TEST RESOURCES USING DUAL SEQUENCES•
}

\author{
Wei Zou ${ }^{1}$, Chris C.N. Chu ${ }^{2}$, Sudhakar M. Reddy ${ }^{1}$, Irith Pomeranz ${ }^{3}$ \\ 'Electrical \& Computer Engineering, University of lowa, Iowa City, IA, 52242, USA \\ ${ }^{2}$ Electrical \& Computer Engineering, Iowa State University, Ames, IA, 50011, USA \\ ${ }^{3}$ Electrical \& Computer Engineering, Purdue University, West Lafayette, IN, 47907, USA
}

\begin{abstract}
In this paper, we propose a new data structure called dual sequences to represent SOC test schedules. Dual sequences are used together with a simulated annealing based procedure to optimize the SOC test application time and tester resources. The problems we consider are generation of optimal test schedules for SOCs and minimizing tester memory and test channels. Results of experiments conducted on ITC' 02 benchmark SOCs show the effectiveness of the proposed method.
\end{abstract}

\section{INTRODUCTION}

As SOC (system on a chip) design moves toward mainstream use, the problem of effectively testing the IP blocks (called cores) within the SOC needs to be addressed. SOC test requires considering the following issues: test access mechanism (TAM) design, core wrapper design, test scheduling, tester memory and tester channels.

TAM is the hardware infrastructure, which transports the test data between the SOC pins and the core wrappers. The core wrapper primarily consists of scan chains placed around the core to isolate the core from its

- The work of W. Zou and S. M. Reddy supported in part by NSF Grant CCR-0097005 and SRC Grant 001-TJ-949. Work of I. Pomeranz supported in part by NSF Grant CCR0098091 and by SRC Grant 2001-TJ-950.

in IFIP International Federation for

Please use the following format when citing this chapter:

Zou, Wei, Chu, Chris, C.N., Reddy, Sudhakar, M., Pomeranz, Irith, 2006, in IFIP International Federation for Information Processing, Volume 200, VLSI-SOC: From Systems to Chips, eds. Glesner, M., Rcis, R., Indrusiak, L., Mooncy, V., Eveking, H., (Boston: Springer), pp. 181-196. 
surrounding logic and serves as an interface between the TAM and the core. A number of approaches ${ }^{1-6,11}$ have been proposed for the core wrapper design.

SOC test scheduling is the procedure of deciding the test start time of every core so as to obtain a minimum test application time for the SOC under certain constraints, such as TAM width (i.e. the number of SOC pins), power dissipation during test, etc.. Since test scheduling depends on the SOC TAM design and the core wrapper design, SOC test requires co-optimization of the TAM, the core wrapper design and the test schedule. Recently a number of works proposed solutions to this problem. Marinissen et al. ${ }^{3}$ presented several methods to design TAMs. Larsson and Peng ${ }^{7,8}$ considered co-optimization of SOC test time and the number of SOC pins under the assumption that a wrapper for each core is given. Chakrabarty ${ }^{9}$ developed an integer linear programming model for minimizing test application time by co-optimization of bandwidth distribution and test bus assignment. Huang et al. ${ }^{10}$ formulated the co-optimization problem as a rectangle packing problem and solved it by using a best-fit heuristic algorithm. To solve the cooptimization problem, a SOC test schedule representation called sequence pair was used together with simulated annealing or heuristics. ${ }^{6,}{ }^{16}$ Other works ${ }^{11-15}$ have investigated the same problem using specialized heuristic procedures.

It was shown that test application time for benchmark SOCs using a simulated annealing algorithm was most often shorter than all earlier proposed heuristic solutions and also shorter than an ILP based procedure when the run time of the ILP procedure was limited (to several hours). ${ }^{6}$ The SOC schedules were represented in the simulated annealing by what are called sequence pairs ${ }^{19}$.

In this paper, we introduce a simple and effective data structure called Dual Sequences (DS) to represent SOC test schedules and use this to obtain optimal SOC test schedules using simulated annealing. Experimental results show that test schedules obtained using DS with simulated annealing are as good as or better than those obtained using sequence pairs while the run time of the simulated annealing procedure is greatly reduced. Another problem we consider is minimization of tester memory and tester channels, again using DS to represent test schedules together with simulated annealing.

\section{THE FEATURES OF SOC TEST TIME}

The core wrapper is the interface between the core and the SOC TAM. It provides several kinds of operation modes, such as normal function, 
interconnect test, bypass test, etc. The test time for a core is derived by the following formula.

$$
\mathrm{T}=\{1+\max (\mathrm{Si}, \mathrm{So})\} * \mathrm{P}+\min (\mathrm{Si}, \mathrm{So})
$$

where $\mathrm{P}$ is the number of test patterns, and Si (So) denotes the length of the longest wrapper scan input (output) chain for the core. The core test time $T$ is decided by the length of the longest wrapper scan chain. So one goal of the core wrapper design is to shorten the longest wrapper scan chain. For this purpose, balanced wrapper design ${ }^{1,11}$ was proposed, which partitions the wrapper scan elements among the wrapper chains to make the length of the wrapper chains as equal as possible.

Next we consider the relationship between the core test time and the core wrapper width. It is known that the test time for a core is a staircase function, which means that there are only some wrapper width values where the core test time changes. These points are called pareto-optimal points. ${ }^{11}$ If the core wrapper is represented by a rectangle with the width representing the wrapper width and the height representing the core test time, there is a set of candidate rectangles for every core corresponding to the paretooptimal core wrapper widths. In co-optimizing wrapper design and SOC test time, one of these rectangles is chosen for each core.

The problem of SOC test scheduling we are considering is stated below.

Given are a SOC with $N$ pins and $N_{c}$ cores. Each core $C_{i}\left(1 \leq i \leq N_{c}\right)$ has a set of $N_{i}$ permissible wrapper configurations. Each wrapper configuration is represented by a pair $\left(W_{i j}, T\left(W_{i j}\right)\right)$, where $W_{i j}$ stands for the width of the $j$-th wrapper configuration for core $C_{i}$ and $T\left(W_{i j}\right)$ stands for the test time of core $\mathrm{C}_{\mathrm{i}}$ with wrapper width $\mathrm{W}_{\mathrm{ij}}$. The objective is to pick one wrapper design for each core, determine the mapping from the SOC pins to the core wrapper pins, and set the test start time for each core such that the SOC test application time is minimized.

This problem can be transformed into the well-known two-dimensional bin packing problem, in which the SOC is represented by a bin with width $\mathrm{N}$ and the set of $N_{i}$ SOC wrappers for every core is represented by a set of $U_{i}$ rectangles with width $\mathrm{W}_{\mathrm{ij}}$ and height $\mathrm{T}\left(\mathrm{W}_{\mathrm{ij}}\right) .{ }^{10}$ The objective is to choose a rectangle for every core $C_{i}$ and pack all the rectangles in the bin, such that height of the bin is minimum.

\section{DUAL SEQUENCES}

In Fig. 1, we illustrate a test schedule for a SOC with six cores. The vertical axis is time and the horizontal axis represents SOC pins. In this schedule, testing of Core 1 , Core 2 and Core 3 starts simultaneously at time $t=0$. Testing of Core 4 is initiated at $t=t_{3}$. Testing of Cores 5 and 6 is 
initiated at $t=t_{4}$. The two parts of Core 5, denoted 5_1 and 5_2, indicate that Core 5 is tested through two non-consecutive subsets of TAM pins. Testing of the SOC is completed at $t_{6}$. As seen from Fig. 1, every test schedule corresponds to a rectangle placement in the bin representing the SOC.

In this section, a new representation called Dual Sequences (DS) is introduced to express the rectangle placement.

The DS for a placement of a set of $\mathbf{n}$ rectangles (cores) is a pair of sequences $(R, W)$, in which $R$ is a sequence of the names of the $\mathrm{n}$ rectangles and $W$ is a sequence of the widths of the $\mathrm{n}$ rectangles listed in $R$. For example, (<R3 R1 R2 R4 >, <4215>) is a DS from which we can see that the placement is composed of four rectangles with widths $4,2,1$ and 5 , respectively. Next we discuss how to represent a rectangle placement by a DS and how to obtain the rectangle placement corresponding to a DS.

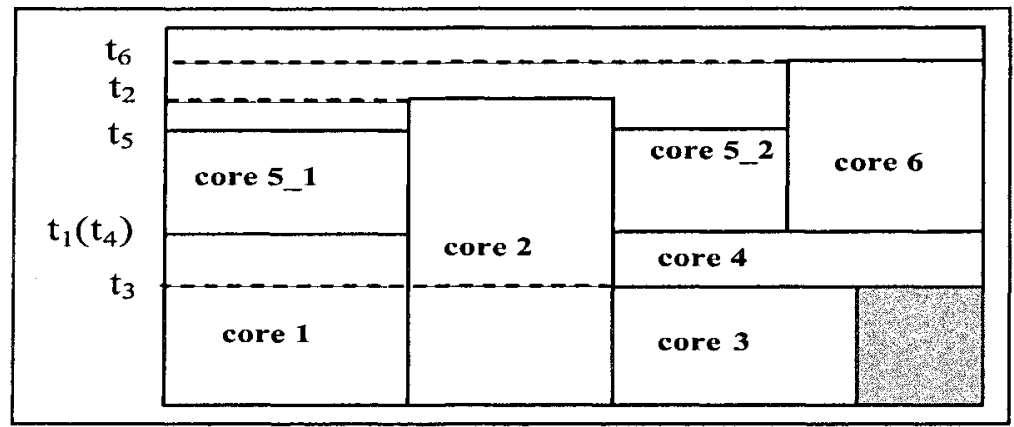

Figure 1. SOC test schedule

\subsection{DS extraction from rectangle placement}

Given a placement of rectangles, the corresponding DS can be obtained by visiting every rectangle in the placement from bottom to top and from left to right. During the visitation, the rectangles we encounter are recorded in $R$ in the order of visiting them and the width corresponding to the discovered rectangles are recorded in $\mathcal{W}$. If a rectangle is split into several sub-rectangles in the placement, the sub-rectangles are merged into one rectangle for representation in $(R, W)$ and its position in $R$ is decided by the first sub rectangle and the width in $W$ is the sum of the widths of the sub-rectangles. A rectangle placement corresponding to a SOC test schedule may have split a rectangle corresponding to a core since its wrapper pins are connected to non-consecutive SOC pins. For example consider the rectangle placement in Fig.1, which has six rectangles R1, R2, R3, R4, R5, R6 corresponding to the 
six cores with widths, say $\Phi 1, \Phi 2, \Phi 3, \Phi 4, \Phi 5$, and $\Phi 6$, respectively. The rectangle $\mathrm{R} 5$ is divided into two sub-rectangles R5_1 and R5_2. As explained next, by visiting each rectangle within the placement and merging the sub-rectangles, we obtain the Dual Sequences ( $<$ R1 R2 R3 R4 R5 R6>, < $\Phi 1 \Phi 2 \Phi 3 \Phi 4 \Phi 5 \Phi 6>$ ). Since testing of R1, R2 and R3 are all scheduled at time zero, they are visited before $R 4$ which is scheduled for testing at $t_{3}$. Within the set of rectangles $R 1, R 2$, and $R 3, R 1$ is visited first since it is left of R2, followed by R2 and then R3. Next R4 is visited. After visiting R4, R5 and $R 6$ are visited but $R 5$ is visited before $R 6$.

\subsection{Mapping from DS to placement of rectangles}

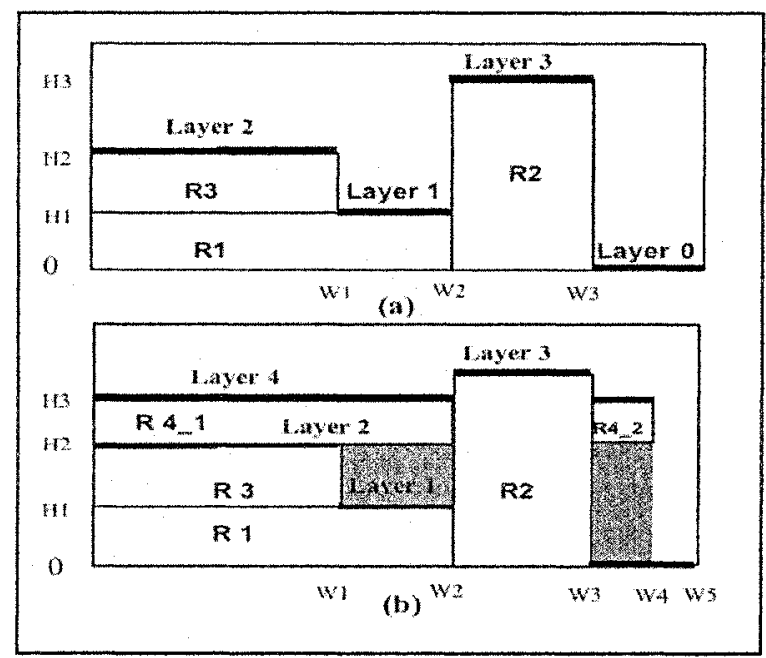

Figure 2. The changes in the layers during bin packing

To obtain a placement from a DS, a greedy algorithm based on two dimensional bin packing is used. The basic idea of this algorithm is that given the sequence $R$ and the width sequence $w$, we pick rectangles from $R$ one at a time in the order of their appearance in $R$ and pack a selected rectangle at a position which is as low as possible (i.e., we schedule the start of the test of the core corresponding to the rectangle as early as possible). It is important to point out the distinction between dual sequences and sequence pairs ${ }^{6,16}$ to represent a bin packing. Given a sequence pairs the corresponding bin packing is uniquely defined and is obtained by a longest path procedure run over two graphs derived from the sequence pairs. The bin packing corresponding to a given DS is not unique. The sequence $R$ 
determines the order in which the rectangles are considered and $\mathcal{W}$ restricts the choice of the width (i.e. wrappers) of the rectangle corresponding to the core being packed. Any procedure to pack the rectangles in the order given by $R$ can be used.

In the proposed method to obtain a rectangle placement from a given DS, a data structure called a layer is used, which corresponds to a position where a yet unplaced rectangle can be placed. A layer has two attributes: starting time and width. The starting time is the height of the layer in the bin and the width indicates the space available at this height. For example, in Fig. 2(a), we have four layers, layer 0 to layer 3 , which are indicated by the thick dark lines. The start time and the width of the layer can be seen from Fig. 2(a). For example, the start time of layerl is $\mathrm{H} 1$ and the width is (W2-W1). Before we describe the procedure to obtain the rectangle placement from a DS, we show how the layers change when a new rectangle is added into an existing partial placement.

Given the partial placement in Fig. 2(a), suppose a new rectangle, say R4 with width (W2+W4-W3) is placed on layer 2. As shown in Fig. 2(b), R4 occupies layer 1, layer 2 and part of layer 0 . Widths of layer 1 and layer 2 are changed to 0 and the width of layer 0 is changed to (W5-W4). A new layer 4 with width (W2+W4-W3) is added. Layer 4 is split into two sublayers as can be seen in Fig. 2(b).

Next we introduce the greedy algorithm for obtaining a rectangle placement from a DS. At the start, there is only one layer whose width is equal to the total TAM width (the number of SOC pins). We pick a rectangle from sequence $R$ from left to right, whose width is decided by the corresponding entry in sequence $W$, and place that rectangle on a layer $\mathrm{L}$, which satisfies the following requirement:

The sum of the width of $L$ and the widths of the layers with non-zero widths whose start time is less than or equal to the start time of layer $\mathrm{L}$ is greater than or equal to the width of the rectangle being placed.

The start time of layer $L$ is the lowest among all layers that satisfy the above requirement.

If there is a power constraint, the core placed at that layer should not violate this constraint.

Following the placement of the rectangle, a new layer with width equal to the width of the just placed rectangle is added to the placement and the widths of the other layers are updated. This procedure is repeated until all the rectangles in sequence $R$ are packed. It should be pointed out that the procedure proposed above is sub-optimal. One reason for this sub-optimality is that when the width of a layer is reduced it may preclude the use of some packing space. For example the shaded area in Fig. 2(b) is not available for future packing after rectangle $\mathrm{R} 4$ is placed. 
Compared to test schedule representation using sequence pairs for obtaining SOC test schedules, the search space for DS is of size $\left(N_{c} ! \times \prod^{N_{0}} K i\right)$, while for the sequence pairs representation the search space is of ${ }^{-1}$ size $\left(\left(N_{c} !\right)^{2} \times \prod^{N c} K i\right)$, where $\mathrm{N}_{c}$ is the number of cores in the SOC and $\mathrm{K}_{\mathrm{i}}$ is the number $b f$ wrapper configurations for core $i$. The size of the search spaces given above is obtained by computing the number of distinct dual sequences and sequence pairs, respectively. Since the search space using DS representation of rectangle placement is smaller, it leads to a much lower run time for test schedule optimization using simulated annealing. As the experiments on ITC' 02 benchmarks reported later show, the optimality of the obtained SOC test schedule is indeed not affected by using dual sequences instead of sequence pairs.

\section{SIMULATED ANNEALING}

Simulated annealing (SA) is a global stochastic optimization algorithm that was first introduced by Kirkpatric et al. ${ }^{17}$. The algorithm begins with an initial solution, and then a neighboring solution is created by perturbing the current solution. If the cost of the neighboring solution is less than that of the current solution, the neighboring solution is accepted; else it is accepted or rejected with some probability. The probability of accepting an inferior solution is a function of a parameter called the temperature. The probability function used is:

$$
p=e^{-\frac{\Delta c}{T}},
$$

where $\Delta \mathrm{C}$ is the change in the cost between the neighboring solution and the current solution and $T$ is the current temperature. The procedure we used to implement the simulated annealing algorithm for finding an optimal SOC test schedule that minimizes the expected test completion time is given below.

Objective: Find an optimal solution $\mathrm{S}_{\mathrm{opt}}$, which makes the cost function $\mathrm{C}\left(\mathrm{S}_{\mathrm{opt}}\right)$ minimum.

Procedure:

1. Construct an initial solution $S_{\text {init }}$;

2. Let the current solution be $S_{\text {cur: }}=S_{\text {init }}$;

3. Set the initial temperature to $\mathrm{T}:=\mathrm{T}_{\text {init; }}$;

4. Set Counter: $=1$;

5. While the stopping criteria is not met do begin

6. While $\mathrm{T}>\mathrm{T}_{\text {final }}$ do begin

7. For $\mathrm{i}:=1$ to Niter do begin

8. Generate a neighboring solution $S_{n}$ from the current solution $S_{\text {cur }}$; 
9. Compute the change in the cost function

$$
\Delta \mathrm{C}=\mathrm{C}\left(\mathrm{S}_{\mathrm{n}}\right)-\mathrm{C}\left(\mathrm{S}_{\mathrm{cur}}\right)
$$

10. If $\Delta C \leq 0$ then $S_{\text {cur }}:=S_{n}$;

11. Else begin

12. $\mathrm{q}=\operatorname{random}(0,1)$;

13. If $\mathrm{q}<e^{\frac{-\Delta c}{T}}$ then $\mathrm{S}_{\mathrm{cur}}:=\mathrm{S}_{\mathrm{n}}$;

14. End

15. End

16. Set new temperature $\mathrm{T}:=\mathrm{K} * \mathrm{~T}$;

17. End

18. Set $\mathrm{T}:=\mathrm{T}_{\text {new; }}$;

19. Counter: $=$ Counter +1 ;

20. End

We use the SA algorithm described above to implement the SOC test scheduling based on dual sequences by specifying the parameters of the SA algorithm as follows.

Cost function $C$ : The height of the bin where the rectangles are placed is defined as the cost function.

Neighboring solution $\mathrm{S}_{\mathrm{n}}$ : A neighboring solution is defined by two types of moves over the dual sequences, given below.

M1: Exchange the position of two randomly chosen rectangles in the first sequence $R$ (note that $W$ is also changed to reflect the exchange in $R$ ).

M2: Change the width (and hence the height) of a rectangle in the sequence $w$ to another allowed width of the rectangle.

During the process of optimization, the probabilities of moves M1 and $\mathrm{M} 2$ are set to 0.5 each.

Initial solution: The initial solution $S_{\text {init }}$ can be set randomly. In order to accelerate the convergence of SA, the test schedule obtained by the heuristic procedure ${ }^{14}$ is used as the initial solution in the experiment reported later.

Initial temperature: The initial temperature $T_{\text {init }}$ is set to 4000 . At the end of each outer loop, temperature $T$ is reset to $T_{\text {new }}=4000+1000 *$ Counter.

Other parameters: These parameters include the final temperature $\mathrm{T}_{\text {final, }}$, the number of iterations Niter at every temperature, the stopping criteria and the temperature reduction multiplier $\mathrm{K}$. In our implementation, these parameters are set as follows.

(1) $\mathrm{T}_{\text {final }}=10$

(2)The number of iterations Niter at each temperature is set to $400 * N_{c}$ where $\mathrm{N}_{\mathrm{c}}$ is the number of rectangles.

(3)The stopping criteria can be decided by the user. In our experiment, if Counter is larger than 10 , the procedure is stopped.

(4) The temperature reduction multiplier $\mathrm{K}$ is set to 0.98 when $\mathrm{T}<10000$; otherwise $\mathrm{K}=0.93$. 


\section{REDUCING ATE RESOURCES}

Automatic Test Equipment (ATE) used in SOC test provides the ability to perform multi-site testing, which allows several copies of a SOC to be tested concurrently. When the number of ATE channels is given, to test a maximum number of SOCs at the same time requires minimization of the TAM width of the SOC while not violating the ATE memory depth constraint (decreasing the TAM width of the SOC will increase the test application time and hence the ATE memory depth requirement). In this section we discuss how the proposed method using DS representation of the test schedules can be used to minimize SOC TAM width as well as the ATE buffer memory depth for a given SOC TAM width.

When an SOC is tested by an ATE, the test channel memory depth required for the $S O C$ is decided by the test data volume. The depth of the test channel memory can be approximated by the SOC test application time (the number of clock cycles). ${ }^{20}$ Therefore, the problem of multi-site SOC test under ATE memory depth constraints can be considered as a problem of reducing the SOC TAM width while the total test application time is fixed. This allows testing of a maximum number of SOCs using a given number of test channels and their buffer memory depth. The SOC multi-site test problem can be solved using the two dimensional bin packing procedure with the width of the bin representing the memory depth constraint and the height of the bin representing the TAM width. We should point out that in the bin packing problem for multi-site testing, a rectangle cannot be divided into several sub-rectangles, which is different from the bin packing problem we discussed before. Dividing rectangles was permitted in the earlier problem since it is not necessary to connect the wrapper pins of a core to adjacent SOC pins. However, in multi-site testing, breaking a rectangle represents interruption of the test of a core, which may not be permitted. A simple way to accommodate the requirement that core tests cannot be interrupted is to require that the new rectangle to be packed must occupy contiguous layers only, thus avoiding division of rectangles.

Another issue that needs to be considered is illustrated by the rectangle packing shown in Fig. 3(a). Fig. 3(a) shows the case where the ATE test channels 1 and 2 are used to test core 2 and ATE test channel 3 is used to test core 1 . Tests for core 1 occupy M1 bits of memory buffer for tester channel 3 and tests for core 2 occupy M2 bits of memory buffer for channels 1 and 2 . Tests for core 3 use all three test channels and hence can only be started after completing the test of core 2 . It should be pointed out that the tests are loaded into the buffer and shifted out to the inputs of the device under test. If the tester architecture is such that all test channel buffers are shifted at the same time and each channel has dedicated memory buffer then 
the buffer bits of channel 3 are don't cares from M1 to M2. However if the tester architecture is such that each test channel is individually controlled, then test channel 3 can be idled after testing core 1 until core 3 test is initiated. In this case the size of the buffer for test channel 3 need only be (M3-M2+M1). The packing shown in Fig. 3(b) for the same cores as in Fig. 3(a) illustrates the situation where the memory buffer contents for test channel 3 is such that don't cares occur only at the end. In this case after the testing of core 1 is complete test channel 3 can be idled. In general, if the packing is such that all the test channel buffers have don't cares only at the end the ATE memory management is simpler. ${ }^{20,}{ }^{21}$ Finally, in some ATE architectures the entire buffer memory can be configured as a single pool of memory that can be dynamically assigned to test channels. ${ }^{21,22}$ For such architectures the total memory requirements for a SOC test is important.

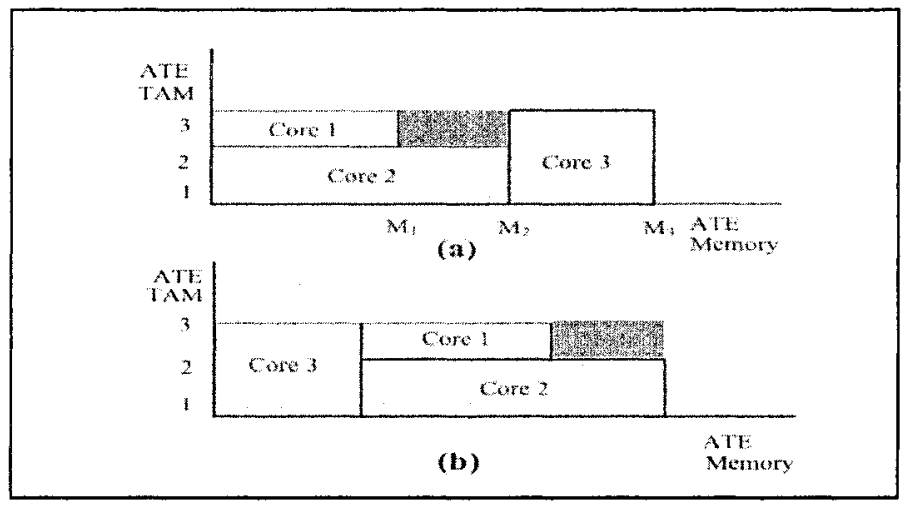

Figure 3. Rectangle packing to optimize ATE resources

For finding SOC test schedules to minimize the number of ATE test channels given the maximum depth of test channel buffers, we used two different procedures to obtain rectangle packings from dual sequences. The first one is a modified version of the procedure ${ }^{20}$ to obtain rectangle packings such that all the don't cares in the memory buffers are at the end. The second procedure is the one described in the last section with the additional constraint that rectangles are not divided during packing.

\section{EXPERIMENTAL RESULTS}

The proposed simulated annealing based algorithm is implemented in $\mathrm{C}++$ and executed on a $\mathrm{PC}$ with a Pentium IV $1.4 \mathrm{GHZ}$ processor and a 512 
MB memory. The implemented procedure was applied to ITC'02 benchmark SOCs ${ }^{18}$ under the assumption of no power constraint.

Table 1. Run times for the proposed method and method of ref. 6

\begin{tabular}{|l|l|lllllll|}
\hline \multirow{2}{*}{ Benchmark } & \multirow{2}{*}{ Method } & \multicolumn{7}{|c|}{ No.SOC pins } \\
& & 32 & 48 & 64 & 80 & 96 & 112 & 128 \\
\hline \multirow{2}{*}{ D695 } & DS & 43.8 & 43.5 & 43.0 & 42.8 & 41.8 & 41.7 & 40.7 \\
& 6 & 52.3 & 54.2 & 56.1 & 56.9 & 58.9 & 61.3 & 67.7 \\
\hline P22810 & DS & 320.7 & 320.9 & 320.3 & 322.8 & 322.9 & 323.1 & 323.9 \\
& 6 & 559.1 & 580.1 & 616.3 & 642.0 & 642.4 & 686.3 & 678.2 \\
\hline \multirow{2}{*}{ P34392 } & DS & 148.6 & 148.0 & 146.2 & 142.2 & 140.0 & 137.7 & 136.1 \\
& 6 & 233.1 & 261.2 & 279.2 & 305.1 & 324.6 & 335.5 & 342.0 \\
\hline \multirow{2}{*}{ P93791 } & DS & 433.2 & 439.0 & 439.2 & 441.9 & 443.4 & 444.2 & 446.2 \\
& 6 & 686.3 & 732.4 & 756.2 & 782.6 & 774.4 & 781.7 & 783.9 \\
\hline \multirow{2}{*}{ G1023 } & DS & 77.6 & 76.8 & 76.0 & 73.7 & 71.8 & 70.3 & 69.1 \\
& 6 & 122.9 & 128.0 & 135.2 & 143.1 & 149.0 & 153.6 & 156.2 \\
\hline \multirow{2}{*}{ U226 } & DS & 26.6 & 27.5 & 26.9 & 26.8 & 26.4 & 26.2 & 26.0 \\
& 6 & 33.3 & 32.2 & 33.3 & 33.7 & 34.4 & 35.3 & 35.4 \\
\hline \multirow{2}{*}{ F2126 } & DS & 12.6 & 11.8 & 11.4 & 11.4 & 11.4 & 11.4 & 11.4 \\
& 6 & 16.1 & 17.2 & 17.5 & 17.4 & 17.4 & 17.4 & 17.4 \\
\hline \multirow{2}{*}{ T512505 } & DS & 330.2 & 357.6 & 354.7 & 359.0 & 362.2 & 356.7 & 348.2 \\
& 6 & 743.5 & 943.9 & 782.0 & 963.4 & 1023.2 & 1038.7 & 1043.4 \\
\hline \multirow{2}{*}{ A586710 } & DS & 24.1 & 23.6 & 23.5 & 23.3 & 23.9 & 22.8 & 23.6 \\
& 6 & 24.4 & 25.2 & 26.4 & 26.1 & 26.2 & 25.7 & 27.4 \\
\hline \multirow{2}{*}{ Q12710 } & DS & 12.3 & 12.3 & 12.3 & 12.3 & 12.3 & 12.3 & 12.3 \\
& 6 & 17.5 & 17.5 & 17.5 & 17.5 & 17.5 & 17.5 & 17.5 \\
\hline \multirow{2}{*}{ D281 } & DS & 27.9 & 27.4 & 26.8 & 26.3 & 25.9 & 25.4 & 25.2 \\
& 6 & 37.0 & 39.5 & 41.3 & 42.8 & 44.3 & 45.1 & 45.4 \\
\hline \multirow{2}{*}{ H953 } & DS & 28.5 & 27.1 & 26.3 & 25.9 & 25.9 & 25.8 & 25.8 \\
& 6 & 49.4 & 52.5 & 53.3 & 53.6 & 53.5 & 53.2 & 53.3 \\
\hline
\end{tabular}

The results of applying the proposed method to SOC test scheduling together with the results reported by earlier methods are reported in Table 2. The proposed simulated annealing based procedure was run for ten iterations and the best schedule obtained is reported. The method used is indicated in column 2, where DS indicates the proposed method and the other methods are indicated by the number of the corresponding reference. The remaining columns give the SOC test application time for the number of SOC pins shown as the heading for the column. The entry for the method(s) achieving the best test application time is shown in bold. The method in ref. 6 also used a simulated annealing algorithm with test schedules represented by sequence pairs and had achieved better schedules than a heuristic method ${ }^{16}$ that also used sequence pairs. For this method also we report the schedule obtained from ten iterations of the procedure. It can be seen that for all the benchmark SOCs, the proposed method achieves better or equal SOC test application time compared to ref. 6 . It can also be observed that the proposed method achieves the same or better test application time than all other methods, 
except in the cases of P93791 with 80 SOC pins and A586710 with 32 SOC pins.

The run times for the proposed simulated annealing based procedure and the earlier procedure ${ }^{6}$ using simulated annealing together with sequence pair are given in Table 1. The run times reported for both procedures are for a total of ten iterations of the procedures. From Table 1 it can be seen that using dual sequences instead of sequence pairs to represent rectangle placements improves the run time of simulated annealing based procedures. A $2 \mathrm{X}$ to $3 \mathrm{X}$ improvement in run time is obtained for most designs.

In Tables 3-6 we report the results on ATE tester channels and buffer memory for four circuits for which data of the earlier work ${ }^{20}$ is available. In the first column we show the maximum memory allowed per test channel. In the next three columns we show the number of tester channels required to deliver the tests using the two procedures described in the last section and the method of ref. 20 , respectively. Procedure DS1 is the proposed simulated annealing based procedure when the don't care bits in the buffer memory of the test channels are all at the end and DS is the procedure where the don't care bits are allowed to be anywhere in the buffer memory. In the next four columns we give the total ATE memory required to store the test input data. For method DS we report two entries. Under DSg we report total memory including the don't cares portion and under DS we report the total memory ignoring the don't care portion. For the other two procedures the don't care portions are not included in the totals reported.

From Tables 3-6 it can be seen that the simulated annealing based procedures require the same or smaller number of test channels compared to the heuristic procedure of ref. 20 for all the SOCs considered. It can also be seen that the total memory required is also smaller for the simulated annealing based procedures.

As an example of how reducing the number of ATE test channels helps reduce the cost of SOC test using multi-site testers, consider a tester with 128 test channels. Note that all SOCs under test can receive the test data simultaneously from the same tester channels. However the test responses from each SOC under test require separate test channels. From Table 4 for SOC p22810 with test channel buffer size limited to $640 \mathrm{~K}$, we note that using methods DS1, DS and of ref. 20, the number of test channels needed to apply tests to all SOCs under test is 12,11 and 13, respectively. However each tested SOC needs the same number of separate test channels to obtain test responses. Thus in this case, the number of SOCs that can be simultaneously tested using a tester with 128 test channels will be 8 using the procedure of ref. 20,9 using procedure DS1 and 10 if procedure DS is used. Thus by using procedure DS the number of SOCs tested per unit of 
time increases by $25 \%$ over the number tested if the procedure from ref. 20 is used.

\section{CONCLUSIONS}

A new data structure called dual sequences to represent rectangle packings is introduced. Using dual sequences together with simulated annealing procedures to obtain optimal SOC test schedules and to reduce ATE test resources were presented. Experimental results on ITC '02 SOC benchmarks showed that the proposed procedures yield better results than procedures proposed earlier.

\section{References}

1. E. J. Marinissen, S. K. Goel and M. Lousberg, Wrapper Design for Embedded Core test, pp.911-920, ITC ,2000.

2. P. Varma and S. Bhatia, A Structured Test Re-Use Methodology for Core-Based System Chips, pp. 294-302, ITC, 1998.

3. E. J. Marinissen, R. Arendsen, G. Bos, H. Dingemanse, M. Lousberg, and C. Wouters, A Structured And Scalable Mechanism for Test Access to Embedded Reusable Cores, pp. 284-293, ITC, 1998.

4. P. T. Gonciari, B. M. Al-Hashimi and N.Nicolici , Addressing Useless Memory in CoreBased System-on-a-Chip Test, pp. 423-430, VTS, 2002.

5. E. J. Marinissen, R. Kapur, and Y. Zorian, On Using IEEE P1500 SECT for Test Plug-nPlay, pp. 770-777, ITC, 2000.

6. W. Zou, S. M. Reddy, I. Pomeranz and Y. Huang, SOC Test Scheduling Using Simulated Annealing, pp. 325-330 VTS, 2003.

7. E. Larsson and Z. Peng, An Integrated System-On-Chip Test Framework, pp. 138-144, DATE, 2001.

8. E. Larsson, Z. Peng and G. Carlsson, The Design and Optimization of SOC Test Solutions, pp. 523-530, ICCAD, 2001.

9. K. Chakrabarty, Design of System-on-Chip Test Access Architectures using Integer Linear Programming, pp. 127-134, VTS, 2000.

10. Y. Huang et. al., Resource Allocation and Test Scheduling for Concurrent Test of Core Based SOC Design, pp. 265-270, ATS, 2001.

11. V. Iyenger, K. Chakrabarty and E. J. Marinssen, Test Wrapper and Test Access Mechanism Co-Optimization for System-on-Chip, pp. 1023-1032, ITC, 2001.

12. V. Iyenger, K. Chakrabarty and E. J. Marinssen, On Using Rectangle Packing for SOC Wrapper/TAM Co-Optimization, pp. 253-258, VTS, 2002.

13. V. Iyengar and K. Chakrabarty and E. J. Marinssen, Integrated Wrapper/TAM CoOptimization, Constraint-Driven Test Scheduling, and Tester Data Volume reduction for SOCs, pp. 685-690, DAC, 2002.

14. Y. Huang et al., Optimal Core Wrapper Width Selection and SOC Test Scheduling Based on3-D Bin Packing Algorithm, pp. 74-82, ITC, 2002.

15. S. K. Goel and E. J. Marinissen, Effective and Efficient Test Architecture Design for SOCs, pp. 529-538, ITC, 2002 . 
16. S. Koranne and V. Iyengar, On the Use of k-tuples for SoC Test Schedule Representation, pp. 539-548, ITC, 2002.

17. S. Kirkpatrick et al, Optimization by Simulated Annealing, pp.671-680, Science, Vol.220, No.4598, 1983.

18. E. J. Marinissen, V. Iyengar and K. Chakrabarty. ITC2002 SOC Benchmarking initiative, http://www.extra.research.philips.com/itc02socbenchm.

19. H. Murata, et. al., VLSI Module Placement Based on Rectangle-Packing by the SequencePair, pp. 1518-1524, IEEE, TCAD, 1996.

20.V. Iyengar et. al., Test resource Optimization for multi-Site testing of SOCs under ATE memory Depth constrains, pp. 1159-1168, ITC, 2002.

21. P. T. Gonciari and B. M. Al-Hashimi, Useless Memory Allocation in System-on-Chip test: Problems and Solutions, pp. 423-429, VTS, 2002.

22. J. Bedsole, R. Raina, A. Crouch and M. S. Abadir, Very Low Cost Tester: Opportunities and Challenges, pp.738-747, ITC, 2001.

Table 2. Test Application Times for ITC'02 SOC Benchmarks

\begin{tabular}{|c|c|c|c|c|c|c|c|c|}
\hline \multirow{2}{*}{ Benchmark } & \multirow{2}{*}{ Method } & \multicolumn{6}{|c|}{ No. SOC pins } & \multirow[b]{2}{*}{$128^{-}$} \\
\hline & & 32 & 48 & 64 & 80 & 96 & 112 & \\
\hline \multirow{5}{*}{ D695 } & DS & 41654 & 28161 & 21025 & 16962 & 14310 & 12134 & 10723 \\
\hline & 6 & 41899 & 28165 & 21258 & 17101 & 14310 & 12134 & 10760 \\
\hline & 11 & 41949 & 28327 & 21423 & 17210 & 16403 & 13023 & 12327 \\
\hline & 12 & 43723 & 30317 & $2302 !$ & 18459 & 15698 & 13415 & 11604 \\
\hline & 15 & 44307 & 28576 & 21518 & 17617 & 14608 & 12462 & 11033 \\
\hline \multirow{4}{*}{ P22810 } & DS & 433403 & 289332 & 219019 & 178402 & 147944 & 128887 & 110940 \\
\hline & 6 & 438619 & 293019 & 219923 & 180004 & 151886 & 132812 & 112515 \\
\hline & 12 & 452639 & 307780 & 246150 & 197293 & 167256 & 145417 & 136941 \\
\hline & 15 & 458068 & 299718 & 222471 & 190995 & 160221 & 145417 & 133405 \\
\hline \multirow{4}{*}{ P34392 } & DS & 960230 & 655607 & 544579 & 544579 & $\mathbf{5 4 4 5 7 9}$ & 544579 & 544579 \\
\hline & 6 & $96 \$ 252$ & 657561 & 544579 & 544579 & 544579 & 544579 & 544579 \\
\hline & 12 & 1023820 & 759427 & 544579 & 544579 & 544579 & 544579 & 544579 \\
\hline & 15 & 1010821 & 680411 & 551778 & 544579 & 544579 & 544579 & 544579 \\
\hline \multirow{5}{*}{ P93791 } & DS & 1763528 & 1175756 & 887619 & 710211 & 594054 & 509845 & 445270 \\
\hline & 6 & 1765797 & 1178397 & 893892 & 718005 & 597182 & 510516 & 451472 \\
\hline & 11 & 1775099 & 1192980 & 899807 & 705164 & 602613 & 521806 & 463707 \\
\hline & 12 & 1851135 & 1248795 & 975016 & 794020 & 627934 & 568436 & 511286 \\
\hline & 15 & 1791638 & 1185434 & 912233 & 718005 & 601450 & 528925 & 455738 \\
\hline \multirow{3}{*}{ G1023 } & DS & 30958 & 21233 & 16048 & 14794 & 14794 & 14794 & 14794 \\
\hline & 6 & 31398 & 21365 & 16067 & 14794 & 14794 & 14794 & 14794 \\
\hline & 15 & 34459 & 22821 & 16855 & 14794 & 14794 & 14794 & 14794 \\
\hline \multirow{3}{*}{ U226 } & DS & 13416 & 10750 & 6746 & 5332 & 5332 & 4080 & 4080 \\
\hline & 6 & 13416 & 10750 & 6746 & 5332 & 5332 & 4080 & 4080 \\
\hline & 15 & 18663 & 13331 & 10665 & 8084 & 7999 & 7999 & 7999 \\
\hline \multirow{3}{*}{ F2126 } & DS & 357088 & 335334 & $\mathbf{3 3 5 3 3 4}$ & 335334 & $\mathbf{3 3 5 3 3 4}$ & 335334 & $\mathbf{3 3 5 3 3 4}$ \\
\hline & 6 & 357088 & 335334 & 335334 & 335334 & 335334 & 335334 & 335334 \\
\hline & 15 & 372125 & 335334 & 335334 & 335334 & 335334 & 335334 & 335334 \\
\hline \multirow{3}{*}{ TS12505 } & DS & 10530995 & 10453470 & 5268868 & 5228420 & 5228420 & 5228420 & 5228420 \\
\hline & 6 & 10530995 & 10453470 & 5268868 & 5228420 & 5228420 & 5228420 & 5228420 \\
\hline & 15 & 10530995 & 10453470 & 5268868 & $\mathbf{5 2 2 8 4 2 0}$ & $\mathbf{5 2 2 8 4 2 0}$ & 5228420 & 5228420 \\
\hline \multirow{3}{*}{ A586710 } & DS & 42198943 & 27785885 & 21343768 & 19041307 & 15031300 & 13401034 & 11486601 \\
\hline & 6 & 42198943 & 27785885 & 21735555 & 19041307 & 15071700 & 14709449 & 12754585 \\
\hline & 15 & 41523868 & 28716501 & 22475033 & 19048835 & 15315476 & 13401034 & 12700205 \\
\hline \multirow{3}{*}{ Q12710 } & DS & 2222349 & 2222349 & 2222349 & 2222349 & 2222349 & 2222349 & 2222349 \\
\hline & 6 & 2222349 & 2222349 & 2222349 & 2222349 & 2222349 & 2222349 & 2222349 \\
\hline & 15 & 2222349 & 2222349 & 2222349 & 2222349 & 2222349 & 2222349 & 2222349 \\
\hline \multirow{3}{*}{ D281 } & DS & 7881 & 5329 & 4070 & 3926 & 3926 & 3926 & 3926 \\
\hline & 6 & 7946 & 5485 & 4070 & 3926 & 3926 & 3926 & 3926 \\
\hline & 15 & 8444 & 6408 & 5084 & 3964 & 3926 & 3926 & 3926 \\
\hline \multirow{3}{*}{ H953 } & DS & 119357 & 119357 & 119357 & 119357 & 119357 & 119357 & 119357 \\
\hline & 6 & 119357 & 119357 & 119357 & 119357 & 119357 & 119357 & 119357 \\
\hline & 15 & 119357 & 119357 & 119357 & 119357 & 119357 & 119357 & 119357 \\
\hline
\end{tabular}


Table 3. SOC g1023: TAM width and ATE memory

\begin{tabular}{|l|l|l|l|l|l|l|l|}
\hline \multirow{2}{*}{ Memory } & \multicolumn{3}{|c|}{ TAM width } & \multicolumn{5}{|c|}{ Total ATE memory } \\
\cline { 2 - 8 } & DSI & DS & 20 & DS1 & DSg & DS & 20 \\
\hline $32 \mathrm{~K}$ & 16 & 16 & 18 & 495679 & 496080 & 495639 & 511464 \\
\hline $40 \mathrm{~K}$ & 13 & 13 & 15 & 495736 & 493047 & 492948 & 505107 \\
\hline $48 \mathrm{~K}$ & 11 & 11 & 13 & 493358 & 494391 & 493717 & 507696 \\
\hline $56 \mathrm{~K}$ & 9 & 9 & 11 & 492936 & 493713 & 492087 & 515956 \\
\hline $64 \mathrm{~K}$ & 8 & 8 & 10 & 493475 & 493404 & 493124 & 514380 \\
\hline $72 \mathrm{~K}$ & 7 & 7 & 9 & 490096 & 490086 & 488965 & 516732 \\
\hline $80 \mathrm{~K}$ & 7 & 7 & 8 & 488759 & 488922 & 488910 & 514538 \\
\hline $88 \mathrm{~K}$ & 6 & 6 & 7 & 489263 & 490291 & 490020 & 506660 \\
\hline $96 \mathrm{~K}$ & 6 & 6 & 6 & 488639 & 490363 & 489495 & 507849 \\
\hline $104 \mathrm{~K}$ & 5 & 5 & 5 & 490060 & 489592 & 489142 & 501840 \\
\hline $112 \mathrm{~K}$ & 5 & 5 & 5 & 488244 & 490029 & 488886 & 500262 \\
\hline $120 \mathrm{~K}$ & 5 & 4 & 5 & 487714 & 489018 & 487870 & 500209 \\
\hline $128 \mathrm{~K}$ & 4 & 4 & 4 & 488271 & 488740 & 488740 & 497167 \\
\hline
\end{tabular}

Table 4. SOC p22810: TAM width and ATE memory

\begin{tabular}{|l|l|l|l|l|l|l|l|}
\hline \multirow{2}{*}{ Memory } & \multicolumn{3}{|c|}{ TAM width } & \multicolumn{5}{c|}{ Total ATE memory } \\
\cline { 2 - 8 } & DS1 & DS & 20 & DS1 & DSg & DS & 20 \\
\hline $256 \mathrm{~K}$ & 30 & 28 & 30 & 7099492 & 7095609 & 6993356 & 7404961 \\
\hline $320 \mathrm{~K}$ & 23 & 22 & 25 & 7027732 & 7003552 & 6967561 & 7134483 \\
\hline $384 \mathrm{~K}$ & 19 & 19 & 21 & 6996904 & 7023324 & 6955179 & 7255983 \\
\hline $448 \mathrm{~K}$ & 17 & 16 & 18 & 7086139 & 6994720 & 6913817 & 7326446 \\
\hline $512 \mathrm{~K}$ & 14 & 14 & 16 & 6926695 & 7096052 & 7008133 & 7350768 \\
\hline $576 \mathrm{~K}$ & 13 & 12 & 14 & 6862197 & 6955836 & 6935482 & 7390568 \\
\hline $640 \mathrm{~K}$ & 12 & 11 & 13 & 6971280 & 6915493 & 6833044 & 7441084 \\
\hline $704 \mathrm{~K}$ & 11 & 10 & 11 & 6804771 & 6886110 & 6820335 & 7234211 \\
\hline $768 \mathrm{~K}$ & 10 & 9 & 11 & 6865817 & 6892560 & 6832015 & 7564350 \\
\hline $832 \mathrm{~K}$ & 9 & 9 & 10 & 6839742 & 6917345 & 6835019 & 7601117 \\
\hline $896 \mathrm{~K}$ & 9 & 8 & 10 & 6797761 & 6932763 & 6823407 & 7784192 \\
\hline $960 \mathrm{~K}$ & 8 & 8 & 9 & 6837474 & 6940258 & 6843692 & 7642819 \\
\hline $1 \mathrm{M}$ & 7 & 7 & 8 & 6935563 & 6897983 & 6810674 & 7245774 \\
\hline
\end{tabular}


Table 5. SOC p93791: TAM width and ATE memory

\begin{tabular}{|l|l|l|l|l|l|l|l|}
\hline \multirow{2}{*}{ Memory } & \multicolumn{3}{|c}{ TAM width } & \multicolumn{5}{|c|}{ Total ATE memory } \\
\cline { 2 - 8 } & DS1 & DS & 20 & DS1 & DSg & DS & 20 \\
\hline $1.00 \mathrm{M}$ & 29 & 29 & 30 & 28801632 & 29165376 & 28701704 & 30569666 \\
\hline $1.256 \mathrm{M}$ & 23 & 23 & 23 & 28365553 & 28977417 & 28673994 & 28853177 \\
\hline $1.512 \mathrm{M}$ & 19 & 19 & 20 & 28416635 & 28749065 & 28485192 & 29587103 \\
\hline $1.768 \mathrm{M}$ & 16 & 16 & 17 & 28350143 & 28881393 & 28598386 & 30209460 \\
\hline $2.000 \mathrm{M}$ & 14 & 14 & 15 & 28448141 & 28576775 & 28221882 & 30570183 \\
\hline $2.256 \mathrm{M}$ & 13 & 13 & 13 & 28235620 & 28732954 & 28267254 & 29108758 \\
\hline $2.512 \mathrm{M}$ & 12 & 12 & 12 & 28304322 & 28902346 & 28223225 & 30385045 \\
\hline $2.768 \mathrm{M}$ & 11 & 11 & 11 & 28184680 & 28196995 & 28026911 & 29499548 \\
\hline $3.000 \mathrm{M}$ & 10 & 10 & 10 & 28157723 & 28665927 & 28160766 & 29635431 \\
\hline $3.256 \mathrm{M}$ & 9 & 9 & 9 & 28227717 & 28570811 & 28100678 & 29121214 \\
\hline $3.512 \mathrm{M}$ & 8 & 8 & 8 & 28056204 & 28417779 & 28194724 & 28853489 \\
\hline $3.768 \mathrm{M}$ & 8 & 8 & 8 & 28211308 & 28699383 & 28096145 & 29038354 \\
\hline $4.000 \mathrm{M}$ & 7 & 7 & 7 & 28301285 & 28420266 & 28189282 & 29096196 \\
\hline
\end{tabular}

Table 6. SOC $\mathrm{p} 34392$ : TAM width and ATE memory

\begin{tabular}{|l|l|l|l|l|l|l|l|}
\hline \multirow{2}{*}{ Memory } & \multicolumn{3}{|c|}{ TAM width } & \multicolumn{5}{|c|}{ Total ATE memory } \\
\cline { 2 - 8 } & DS1 & DS & 20 & DS1 & DSg & DS & 20 \\
\hline $768 \mathrm{~K}$ & 21 & 21 & 23 & 15499573 & 15572458 & 15509657 & 15975513 \\
\hline $896 \mathrm{~K}$ & 18 & 18 & 20 & 16037390 & 15381530 & 15348449 & 16676762 \\
\hline $1.00 \mathrm{M}$ & 16 & 15 & 16 & 15506240 & 15221266 & 15194202 & 15645989 \\
\hline $1.128 \mathrm{M}$ & 14 & 14 & 15 & 15399516 & 15267172 & 15213829 & 16227655 \\
\hline $1.256 \mathrm{M}$ & 13 & 12 & 14 & 15286752 & 15224186 & 15152249 & 15961051 \\
\hline $1.384 \mathrm{M}$ & 11 & 11 & 13 & 15336206 & 15345128 & 15268571 & 16713779 \\
\hline $1.512 \mathrm{M}$ & 11 & 10 & 12 & 15239519 & 15228804 & 15179270 & 15910317 \\
\hline $1.640 \mathrm{M}$ & 10 & 10 & 11 & 15177894 & 15304504 & 15165987 & 15474763 \\
\hline $1.768 \mathrm{M}$ & 9 & 9 & 10 & 15132399 & 15179765 & 15097375 & 15890652 \\
\hline $1.896 \mathrm{M}$ & 8 & 8 & 10 & 15124570 & 15176594 & 15127484 & 16330357 \\
\hline $2.000 \mathrm{M}$ & 8 & 8 & 9 & 15114833 & 15180006 & 15080645 & 16588577 \\
\hline
\end{tabular}

